\title{
Comparative study of ordered and disordered $\mathrm{Y}_{1-x} \mathrm{Sr}_{x} \mathrm{CoO}_{3-\delta}$
}

\author{
S. Fukushima * T. Sato, D. Akahoshi, and H. Kuwahara \\ Department of Physics, Sophia University \\ Chiyoda-ku, Tokyo 102-8554, JAPAN
}

\begin{abstract}
We have succeeded in preparing $A$-site ordered- and disordered- $\mathrm{Y}_{1 / 4} \mathrm{Sr}_{3 / 4} \mathrm{CoO}_{3-\delta}$ with various oxygen deficiencies $\delta$, and have made comparative study of their structural and physical properties. In the $A$-site ordered structure, oxygen vacancies order, and $\delta=0.34$ sample shows a weak ferromagnetic transition beyond $300 \mathrm{~K}$. On the other hand, in the $A$-site disordered structure, no oxygen vacancy ordering is observed, and $\delta=0.16$ sample shows a ferromagnetic metallic transition around $150 \mathrm{~K}$. $A$-site disordering destroys the orderings of oxygen-vacancies and orbitals, leading to the strong modification of the electronic phases.
\end{abstract}

PACS numbers: $75.30 .-\mathrm{m}$

Cobalt oxides with perovskite-based structure exhibit many attracting phenomena such as ferromagnetism, metal-insulator transition, and spin-state transition. These phenomena are caused by the various spin states of Co ion such as low-spin (LS), intermediatespin (IS), and high-spin (HS) states. $\mathrm{La}_{1-x} \mathrm{Sr}_{x} \mathrm{CoO}_{3}$ is the most typical example: The ground state of $x=0$ compound is a nonmagnetic insulator with LS state of $\mathrm{Co}^{3+}$. [1, 2] With increase of Sr content $x$, a ferromagnetic metallic state emerges in $x \geq 0.18$, which is driven by the double-exchange interaction between $\mathrm{Co}^{4+}$ in $\mathrm{LS}$ state and $\mathrm{Co}^{3+}$ in IS state. [3, 4]

Among many perovskite cobaltites, $\mathrm{Y}_{1-x} \mathrm{Sr}_{x} \mathrm{CoO}_{3-\delta}$ attracts our interest, because of its characteristic structural features and high magnetic-transition temperatures. $A$-site ordered- $\mathrm{Y}_{1-x} \mathrm{Sr}_{x} \mathrm{CoO}_{3-\delta}$ has been first reported by Withers et al. [5] and Istomin et al. [6] This compound has large oxygen deficiency $\delta$. The structure of ordered- $\mathrm{Y}_{1 / 4} \mathrm{Sr}_{3 / 4} \mathrm{CoO}_{2.66}$ can be regarded as the alternate stacking of $A \mathrm{O}$ and $\mathrm{CoO}_{1.7}$ sheets, where $A$ site cations and oxygen vacancies concomitantly order as shown in Fig. 1(a). $\mathrm{Y}_{1 / 4} \mathrm{Sr}_{3 / 4} \mathrm{O}$ sheet stacks along the $c$ axis with translation $\langle 1 / 2,1 / 2,0\rangle$. $\mathrm{CoO}_{1.3}$ and $\mathrm{CoO}_{2}$ sheets alternately stack along the $c$-axis with translation $\langle 1 / 2,1 / 2,0\rangle$ to form the four times periodicity. For $0.75 \leq x \leq 0.8, A$-site ordered- $\mathrm{Y}_{1-x} \mathrm{Sr}_{x} \mathrm{CoO}_{3-\delta}$ undergoes a weak ferromagnetic transition at $335 \mathrm{~K}$. 7] Ishiwata et al. propose that the weak ferromagnetism is related to the orbital ordering. [8] In addition, the physical properties are sensitive to the variation of oxygen deficiency $\delta$. For example, in $x=0.33$, the ground state is transformed from an antiferromagnetic insulator to a ferromagnetic metal with a slight decrease of $\delta$. 9]

The high magnetic transition temperature is due to the $A$-site ordered structure free from chemical disorder. The $A$-site arrangement in perovskite-based oxides plays an important role in determining their physical properties. 10] In $A$-site ordered- $R_{1 / 2} \mathrm{Ba}_{1 / 2} \mathrm{MnO}_{3}(R=$ rare earth ion), ferromagnetic or charge-ordering transi-

*Electronic address: fukush-s@sophia.ac.jp (a)

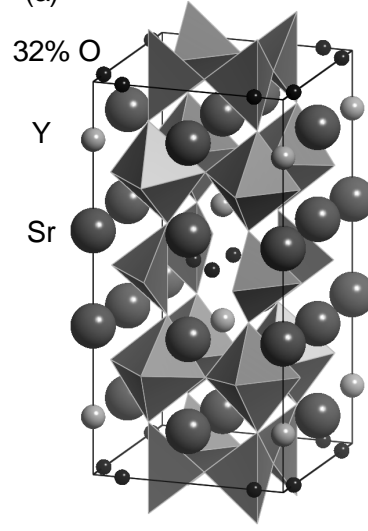

ordered $-\mathrm{Y}_{1 / 4} \mathrm{Sr}_{3 / 4} \mathrm{CoO}_{2.66}$ (b)

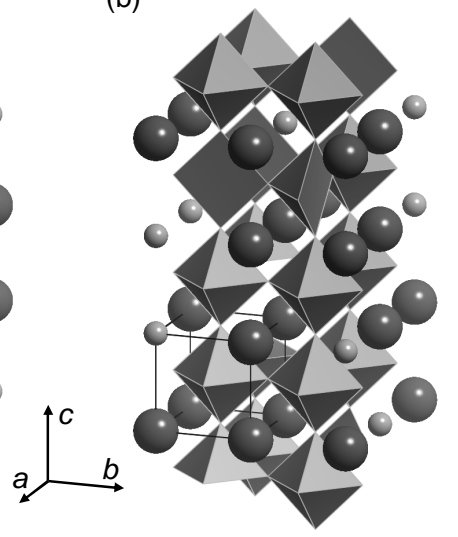

disordered $-\mathrm{Y}_{1 / 4} \mathrm{Sr}_{3 / 4} \mathrm{CoO}_{2.84}$
FIG. 1: Schematic structure of (a) $A$-site ordered$\mathrm{Y}_{1 / 4} \mathrm{Sr}_{3 / 4} \mathrm{CoO}_{2.66}$ and (b) disordered- $\mathrm{Y}_{1 / 4} \mathrm{Sr}_{3 / 4} \mathrm{CoO}_{2.84}$. Small dark spheres in (a) represent oxygen site $30 \%$ occupied. The ordered- $\mathrm{Y}_{1 / 4} \mathrm{Sr}_{3 / 4} \mathrm{CoO}_{2.66}$ has $\mathrm{CoO}_{2}$ layers at $z=$ $1 / 4$ and $3 / 4$, and $\mathrm{CoO}_{1.3}$ layers at $z=0$ and $1 / 2$ with translation $\langle 1 / 2,1 / 2,0\rangle$. In disordered- $\mathrm{Y}_{1 / 4} \mathrm{Sr}_{3 / 4} \mathrm{CoO}_{2.84}, \mathrm{Y}^{3+}$ and $\mathrm{Sr}^{2+}$ occupy $A$-site at random, and oxygen vacancies are randomly distributed.

tions exceed room temperature, and a bicritical feature can be seen in the phase diagram. The random potential arising from $A$-site disordering suppresses long-range orders, and gives rise to the colossal magnetoresistive state near the bicritical region. In the cobalt oxide system just like the manganese oxide system, the $A$-site arrangement probably affects the physical properties through Co spin state and orbital arrangement. However, there are few reports on randomness effect of $A$-site ions in the cobalt oxides. [11] In this study, we prepared $A$-site ordered- and disordered- $\mathrm{Y}_{1 / 4} \mathrm{Sr}_{3 / 4} \mathrm{CoO}_{3-\delta}$ with various $\delta$ and have made a comparative study of them in order to investigate the $A$-site randomness effect on their structural and physical properties.

$A$-site ordered- and disordered- $\mathrm{Y}_{1 / 4} \mathrm{Sr}_{3 / 4} \mathrm{CoO}_{3-\delta}$ were prepared in a polycrystalline form by solid state reaction. Mixed powders of $\mathrm{Y}_{2} \mathrm{O}_{3}, \mathrm{SrCO}_{3}$, and $\mathrm{CoO}$ were 
heated at $1073 \mathrm{~K}$ in air with a few intermediate grindings, and sintered at $1423 \mathrm{~K}$ in air. Then the sample was annealed at $1173 \mathrm{~K}$ in Ar atmosphere. The resulting product has $A$-site ordered form. On the other hand, $A$-site disordered form is obtained by quenching the sample from 1473 to $77 \mathrm{~K}$. We prepared orderedand disordered- $\mathrm{Y}_{1 / 4} \mathrm{Sr}_{3 / 4} \mathrm{CoO}_{3-\delta}$ with various $\delta$ through different annealing conditions. The detailed conditions will be described elsewhere. The values of $\delta$ were determined through iodometric titration with an accuracy of \pm 0.01 . The crystallographic analysis of the obtained samples was performed by X-ray-diffraction (XRD) method at room temperature. The resistivity was measured by a standard four-probe method from 5 to $350 \mathrm{~K}$. The magnetic properties were measured using a Quantum Design, Physical Property Measurement System (PPMS) from 5 to $350 \mathrm{~K}$.

From the XRD measurements, we confirmed that $\mathrm{Y}_{1 / 4} \mathrm{Sr}_{3 / 4} \mathrm{CoO}_{2.66}$ annealed in $\mathrm{Ar}$ has the $A$-site ordered structure as shown in Fig. 1(a). $A$-site ordered$\mathrm{Y}_{1 / 4} \mathrm{Sr}_{3 / 4} \mathrm{CoO}_{3-\delta}$ with $\delta=0.44$ and 0.30 have the similar $A$-site ordered structure as that of $\delta=0.34$ sample, but the arrangement of oxygen vacancies are different from each other. On the other hand, quenched $\mathrm{Y}_{1 / 4} \mathrm{Sr}_{3 / 4} \mathrm{CoO}_{3-\delta}(0.15 \leq \delta \leq 0.27)$ samples have a simple cubic perovskite structure. Bragg peaks arising from $A$-site and/or oxygen-vacancy orderings are not observed, indicating that $\mathrm{Y}^{3+}$ and $\mathrm{Sr}^{2+}$ randomly occupy $A$-site, and that oxygen vacancies are randomly distributed as shown in Fig. 1(b). These results indicate that $A$-site disordering makes the oxygen-vacancy order destabilized. The detailed results of the structural properties will be published elsewhere.

Figure 2(a) shows the temperature dependence of the magnetization $(M-T)$ of $A$-site ordered$\mathrm{Y}_{1 / 4} \mathrm{Sr}_{3 / 4} \mathrm{CoO}_{3-\delta}$. The magnetization of $\delta=0.34$ shows an abrupt increase below $330 \mathrm{~K}$. The onset of the magnetization corresponds to the weak ferromagnetic transition. The magnetic field dependence of the magnetization $(M-H)$ shows a ferromagnetic behavior at $300 \mathrm{~K}$, but the magnetization remains unsaturated even at 80 $\mathrm{kOe}\left(M=0.19 \mu_{\mathrm{B}} / \mathrm{Co}\right)$. This magnetic behavior is consistent with that reported by Kobayashi et al. [7]. On the basis of structural refinement, they propose two models for the magnetic order. One is a canted antiferromagnetic state, and the other is a ferrimagnetic state due to the antiferromagnetic coupling between the HS and IS states of $\mathrm{Co}^{3+}$.88] Slight increase or decrease of $\delta$ from 0.34 suppresses the weak ferromagnetic phase around $325 \mathrm{~K}$. The magnetization of $\delta=0.30$ gradually increases below $250 \mathrm{~K}$, and shows weak ferromagnetic behavior at low temperatures. On the other hand, $\delta=0.44$ shows a magnetic transition around $230 \mathrm{~K}$. The magnetization of $\delta=0.44$ sample linearly depends on applied magnetic fields at $5 \mathrm{~K}$, while the $M-H$ curves of $\delta=0.34$ and 0.30 indicate that they have ferromagnetic components. With decrease of $\delta$ from 0.44 , that is, with increase of Co valence, the ferromagnetic component tends to increase at

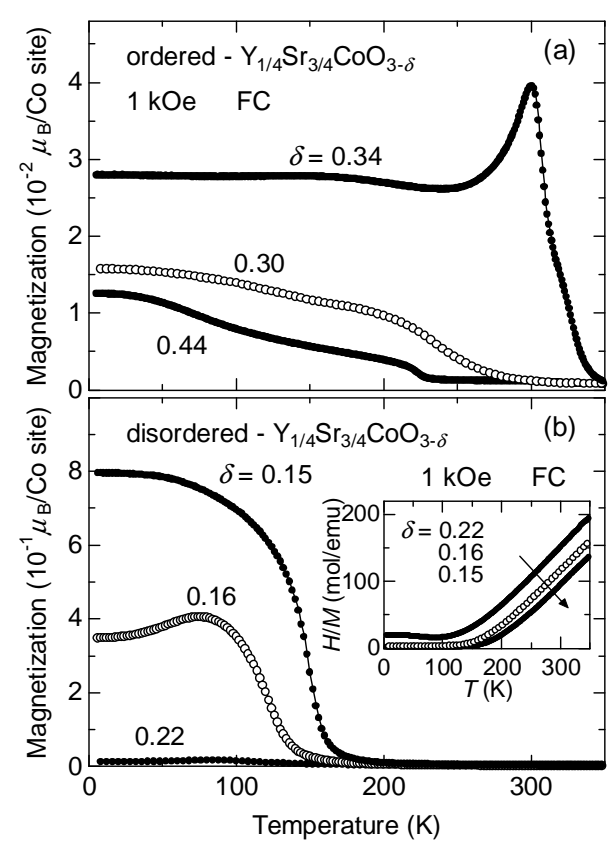

FIG. 2: Temperature dependence of the magnetization of (a) $A$-site ordered- $\mathrm{Y}_{1 / 4} \mathrm{Sr}_{3 / 4} \mathrm{CoO}_{3-\delta}(\delta=0.30,0.34$, and 0.44$)$ and (b) disordered- $\mathrm{Y}_{1 / 4} \mathrm{Sr}_{3 / 4} \mathrm{CoO}_{3-\delta}(\delta=0.15,0.16$, and $0.22)$. The inset shows the inverse susceptibility of $A$-site disordered- $\mathrm{Y}_{1 / 4} \mathrm{Sr}_{3 / 4} \mathrm{CoO}_{3-\delta}$.

low temperatures. The magnetization of $\delta=0.44,0.34$, and 0.30 at $80 \mathrm{kOe}$ are $0.127,0.132$, and $0.23 \mu_{\mathrm{B}} / \mathrm{Co}$, respectively.

Figure 2(b) shows the $M-T$ curves of $A$-site disordered$\mathrm{Y}_{1 / 4} \mathrm{Sr}_{3 / 4} \mathrm{CoO}_{3-\delta}$. The magnetization of $\delta=0.15$ exhibits an abrupt jump around $150 \mathrm{~K}$. The $M-H$ curve of $\delta=0.15$ shows the typical ferromagnetic hysteresis below $150 \mathrm{~K}$, in contrast to that of ordered- $\mathrm{Y}_{1 / 4} \mathrm{Sr}_{3 / 4} \mathrm{CoO}_{2.66}$. The saturation magnetization of $\delta=0.15$ at $5 \mathrm{~K}$ is 1.2 $\mu_{\mathrm{B}} /$ Co. With increase of $\delta$, the ferromagnetic phase is drastically suppressed. The magnetic transition temperatures of $A$-site disordered- $\mathrm{Y}_{1 / 4} \mathrm{Sr}_{3 / 4} \mathrm{CoO}_{3-\delta}$ are much lower than those of $A$-site ordered- $\mathrm{Y}_{1 / 4} \mathrm{Sr}_{3 / 4} \mathrm{CoO}_{3-\delta}$. As shown in the inset of Fig. 2(b), the temperature dependence of inverse susceptibility $(H / M-T)$ of disordered$\mathrm{Y}_{1 / 4} \mathrm{Sr}_{3 / 4} \mathrm{CoO}_{3-\delta}(\delta=0.15,0.16$, and 0.22$)$ obeys CurieWeiss law above $250 \mathrm{~K}$ with Weiss temperatures $\theta=$ $190.3,169.1$, and $134.1 \mathrm{~K}$, and effective moments estimated from the observed slopes in this region are $P_{\text {eff }}$ $=3.06,3.02$, and $3.00 \mu_{\mathrm{B}} / \mathrm{Co}$, respectively. This result indicates that magnetic interaction among Co-spins is ferromagnetic for disordered- $\mathrm{Y}_{1 / 4} \mathrm{Sr}_{3 / 4} \mathrm{CoO}_{3-\delta}$ with $\delta=$ 0.15 to 0.22 .

Figure 3(a) shows the temperature dependence of the resistivity $(\rho-T)$ of $A$-site ordered- $\mathrm{Y}_{1 / 4} \mathrm{Sr}_{3 / 4} \mathrm{CoO}_{3-\delta}$. The resistivities of all the samples show semiconducting or insulating behavior. With decrease of $\delta$, the resistivity abruptly drops over the whole temperatures. Taking into account the results of the $M-T$ curves, this implies 


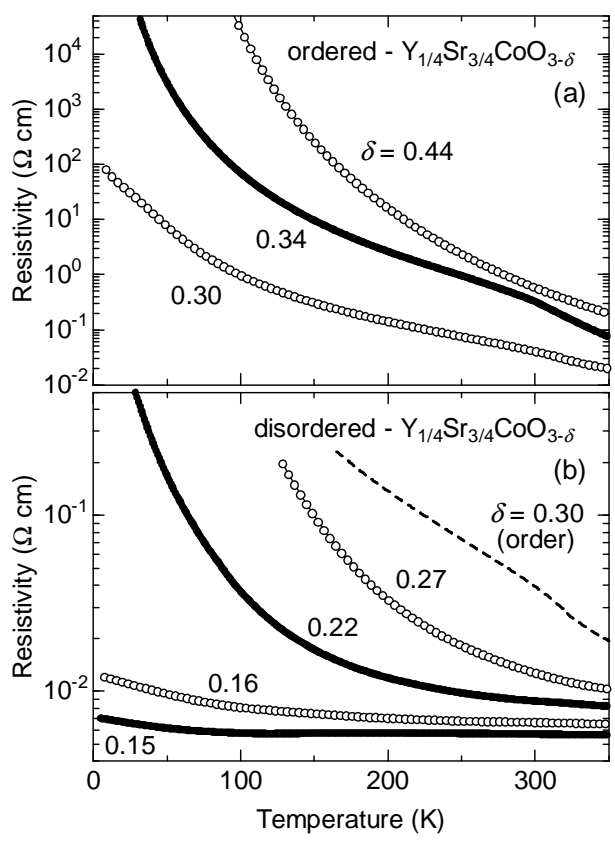

FIG. 3: Temperature dependence of the resistivity of (a) $A$ site ordered- $\mathrm{Y}_{1 / 4} \mathrm{Sr}_{3 / 4} \mathrm{CoO}_{3-\delta}(\delta=0.30,0.34$, and 0.44$)$ and (b) disordered- $\mathrm{Y}_{1 / 4} \mathrm{Sr}_{3 / 4} \mathrm{CoO}_{3-\delta}(\delta=0.15,0.16,0.22$, and $0.27)$. The resistivity of ordered- $\mathrm{Y}_{1 / 4} \mathrm{Sr}_{3 / 4} \mathrm{CoO}_{2.70}$ is also shown (broken line) for comparison.

the increase of ferromagnetic metallic components except for $\delta=0.34$ having large ferromagnetic component. $\delta$ $=0.34$ shows a clear kink around $300 \mathrm{~K}$, which corresponds to the enhanced weak ferromagnetic transition. Figure 3(b) shows the $\rho$ - $T$ curves of $A$-site disordered$\mathrm{Y}_{1 / 4} \mathrm{Sr}_{3 / 4} \mathrm{CoO}_{3-\delta} . \quad \delta=0.27$ shows insulating behavior. With decrease of $\delta$, the resistivity is steeply decreased similar to the case of $A$-site ordered- $\mathrm{Y}_{1 / 4} \mathrm{Sr}_{3 / 4} \mathrm{CoO}_{3-\delta}$, and $\delta=0.15$ sample shows almost metallic behavior.

Then, we will discuss the effect of $A$-site disordering on the structural properties of $\mathrm{Y}_{1 / 4} \mathrm{Sr}_{3 / 4} \mathrm{CoO}_{3-\delta}$. In the $A$-site ordered structure $(0.30 \leq \delta \leq 0.44)$, the periodic-potential and lattice-distortion associated with $\mathrm{Y} / \mathrm{Sr}$ ordering are likely to stabilize the oxygenvacancy order and orbital order. In contrast, in $A$ - site disordered- $\mathrm{Y}_{1 / 4} \mathrm{Sr}_{3 / 4} \mathrm{CoO}_{3-\delta}(0.15 \leq \delta \leq 0.27)$, the random-potential and lattice-distortion due to $\mathrm{Y} / \mathrm{Sr}$ disordering destroy the oxygen-vacancy order.

Such randomness strongly affects the physical properties as well as the structural properties. As shown in Fig. 2, the magnetic transition temperatures of $A$ site disordered- $\mathrm{Y}_{1 / 4} \mathrm{Sr}_{3 / 4} \mathrm{CoO}_{3-\delta}$ are much lower than those of ordered- $\mathrm{Y}_{1 / 4} \mathrm{Sr}_{3 / 4} \mathrm{CoO}_{3-\delta}$. Furthermore, a deviation of $\delta$ from 0.34 as well as $A$-site disordering has much effect on the magnetic structure. In $A$-site ordered$\mathrm{Y}_{1 / 4} \mathrm{Sr}_{3 / 4} \mathrm{CoO}_{3-\delta}, \delta=0.34$ shows the highest magnetic transition temperature (the weak ferromagnetic transition at $330 \mathrm{~K}$ ). As described above, the oxygen-vacancy ordered structure depends on $\delta$. The oxygen-vacancy ordering pattern near $\delta=0.34$ may be essential for the weak ferromagnetism. The relation between the arrangement of oxygen-vacancy and the magnetic structure is now under investigation. The magnetic and transport properties of $A$-site disordered- $\mathrm{Y}_{1 / 4} \mathrm{Sr}_{3 / 4} \mathrm{CoO}_{3-\delta}$ resemble those of $\mathrm{La}_{1-x} \mathrm{Sr}_{x} \mathrm{CoO}_{3}$. [3] In $\mathrm{La}_{1-x} \mathrm{Sr}_{x} \mathrm{CoO}_{3}$, the magnetization and the magnetic transition temperature increase continuously with increasing Sr content $x$ or Co valence, and the ferromagnetic metallic state is dominant for $x \geq 0.18$. The ferromagnetic metallic state of $A$-site disordered- $\mathrm{Y}_{1 / 4} \mathrm{Sr}_{3 / 4} \mathrm{CoO}_{3-\delta}$ is likely to be attributed to double-exchange interaction just like the case of $\mathrm{La}_{1-x} \mathrm{Sr}_{x} \mathrm{CoO}_{3} \cdot[3]$

In summary, we have prepared $A$-site ordered- and disordered- $\mathrm{Y}_{1 / 4} \mathrm{Sr}_{3 / 4} \mathrm{CoO}_{3-\delta}$, and have investigated their structural and physical properties. Oxygen vacancy orders in the $A$-site ordered structure, but not in the $A$-site disordered structure. $A$-site disordered$\mathrm{Y}_{1 / 4} \mathrm{Sr}_{3 / 4} \mathrm{CoO}_{3-\delta}$ exhibits the magnetic transition below $150 \mathrm{~K}$, which is quite lower than those of ordered$\mathrm{Y}_{1 / 4} \mathrm{Sr}_{3 / 4} \mathrm{CoO}_{3-\delta}$. The $A$-site randomness suppresses magnetic order in cobalt oxide $\mathrm{Y}_{1 / 4} \mathrm{Sr}_{3 / 4} \mathrm{CoO}_{3-\delta}$ as reported in manganese oxide $R_{1 / 2} \mathrm{Ba}_{1 / 2} \mathrm{MnO}_{3}$. [10]

This work was supported by Iketani Science and Technology Foundation, the Matsuda Foundation, the Asahi Glass Foundation, and by Grant-in-Aid for Scientific Research on Priority Areas (No.451) from the Ministry of Education, Culture, Sports, Science and Technology (MEXT), Japan.
[1] M. A. Senaris-Rodriguez and J. B. Goodenough, J. Solid State Chem. 116, 224 (1995).

[2] K. Asai, A. Yoneda, O. Yokokura, J. M. Tranquada, G. Shirane, and K. Kohn, J. Phys. Soc. Jpn. 67, 290 (1998).

[3] M. Itoh, I. Natori, S. Kubota, and K. Motoya, J. Phys. Soc. Jpn. 63, 1486 (1994).

[4] M. A. Senaris-Rodriguez and J. B. Goodenough, J. Solid State Chem. 118, 323 (1995).

[5] R. L. Withers, M. James, and D. J. Goossens, J. Solid State Chem. 174, 198 (2003).

[6] S. Ya. Istomin, J. Grins, G. Svensson, O. A. Drozhzhin, V. L. Kozhevnikov, E.V. Antipov, and J. P. Attfield, Chem. Mater. 15, 4012 (2003).
[7] W. Kobayashi, S. Ishiwata, I. Terasaki, M. Takano, I. Grigoraviciute, H. Yamauchi, and M. Karppinen, Phys. Rev. B 72, 104408 (2005).

[8] S. Ishiwata, W. Kobayashi, I. Terasaki, K. Kato, and M. Takata, Phys. Rev. B 75, 220406(R) (2007).

[9] A. Maignan, S.Hébert, V. Caignaert, V. Pralong, and D. Pelloquin, J. Solid State Chem. 178, 868 (2005).

[10] D. Akahoshi, M. Uchida, Y. Tomioka, T. Arima, Y. Matsui, and Y. Tokura, Phys. Rev. Lett. 90, 177203 (2003).

[11] T. Nakajima, M. Ichihara, and Y. Ueda, J. Phys. Soc. Jpn. 74, 1572 (2005). 